\title{
Automatic Target and Trajectory Identification for Deep Brain Stimulation (DBS) Procedures
}

\author{
Ting Guo ${ }^{1,3}$, Andrew G. Parrent ${ }^{2}$, and Terry M. Peters ${ }^{1,3}$ \\ ${ }^{1}$ Robarts Research Institute and University of Western Ontario \\ ${ }^{2}$ The London Health Sciences Centre, Department of Neurosurgery \\ London, Ontario, Canada N6A 5K8 \\ ${ }^{3}$ Biomedical Engineering Graduate Program, University of Western Ontario \\ London, Ontario, Canada N6A 5B9 \\ \{tguo, tpeters\} @imaging.robarts.ca
}

\begin{abstract}
This paper presents an automatic surgical target and trajectory identification technique for planning deep brain stimulation (DBS) procedures. The probabilistic functional maps, constructed from population-based actual stimulating field information and intra-operative electrophysiological activities, were integrated into a neurosurgical visualization and navigation system to facilitate the surgical planning and guidance. In our preliminary studies, we compared the actual surgical target locations and trajectories established by an experienced stereotactic neurosurgeon with those automatically planned using our probabilistic functional maps on 10 subthalamic nucleus (STN) DBS procedures. The average displacement between the surgical target locations in both groups was $1.82 \mathrm{~mm}$ with a standard deviation of $0.77 \mathrm{~mm}$. The difference between the surgical trajectories was $3.1^{\circ}$ and $2.3^{\circ}$ in the lateral-to-medial and anterior-to-posterior orientations respectively.
\end{abstract}

\section{Introduction}

Deep brain stimulation (DBS) eliminates abnormally patterned activity from certain nuclei by implanting a multi-electrode stimulator into a specific midbrain structure where continuous high frequency electrical stimulation generated by a neuropacemaker is delivered [1]. Accurate implantation of the stimulator is critical to achieve the optimal surgical outcome, because small deviations in the electrode positioning may cause muscle contraction, speech and language disorders, ocular deviation, and visual defects $[1,2]$. In clinical practice, due to the inadequacy of the anatomical and functional information available pre-operatively, invasive electrophysiological measurements must be obtained intra-operatively with multiple exploratory electrodes to facilitate the mapping of functionally distinct deep brain structures and the identification of the optimal surgical target [1].

In addition to direct targeting on standard pre-operative medical images, rigid or non-rigid alignment of the digitized stereotactic atlases [3] with patient-specific preoperative brain images is often utilized to assist the target identification. However the inherent limitations of current anatomical brain atlases may limit their applicability. Recently, Yelnik et al described a 3D deformable histological atlas of the human 
basal ganglia [4] that contained functional information derived from immunohistochemical studies. In addition, functional atlases [5,6] and databases [7] containing intra-operatively acquired subcortical electrophysiology from a number of patients have been implemented to complement the anatomical and histological atlases. Precise localization of the target is more likely to be achieved with the assistance of pre-operatively available electrophysiological information [7].

This paper focuses on constructing 3D probabilistic maps of functional data and integrating these maps into our neurosurgical system to accomplish automatic target and trajectory identification for DBS procedures. The optimal trajectory is identified with a probabilistic approach that maximizes the possibility of treatment efficacy while minimizing that of side effects. Preliminary studies were conducted to evaluate the effectiveness of this technique in surgical target and trajectory identification for subthalamic nucleus (STN) DBS procedures. Representing spatial distribution of population-based actual target stimulation information and deep-brain electrophysiological activities, novel probabilistic maps incorporated into our neurosurgical system, after non-rigidly aligned to a patient brain space prior to surgery, can provide robust and accurate automatic initialization of the surgical target and trajectory.

\section{Materials and Methods}

\subsection{Image Registration}

Frame-to-Image: The image-to-patient registration is achieved with a $0.5 \mathrm{~mm}$ fiducial localization error (FLE) using an automatic frame-to-image registration procedure.

Post-operative-to-Pre-operative: The post- and pre-operative images are registered using a rigid registration that maximizes a cross-correlation metric to map the final DBS electrode placement information from the post- to the pre-operative image.

Functional Data Mapping: We employ a fast and completely unsupervised multiresolution registration approach, AtamaiWarp [8], to generate the three-dimensional transformation matrix and warp grid describing the voxel to voxel mapping between each patient pre-operative image and a standard brain template.

\subsection{Data Preparation}

MR image acquisition: The pre- and post-operative MR images of 52 STN DBS procedures (26 left and 26 right) were acquired with a 3D SPGR sequence on a $1.5 \mathrm{~T}$ GE Signa scanner (TR/TE $8.9 / 1.9 \mathrm{~ms}$, FA $20^{\circ}$, voxel size $1.17 \times 1.17 \times 1 \mathrm{~mm}^{3}$, in-slice resolution $256 \times 256$ ).

Electrode and stimulation field modeling: A B-spline based method allows a smooth contour of the DBS electrode (Medtronic 3389, Medtronic Inc., Minneapolis, MN, USA) on the post-operative image to be created with user defined control points. Theoretically, a patient specific electrical stimulation model [9] considering the tissue conductivity properties would describe the stimulation field distribution more accurately, however due to the unavailability of diffusion tensor imaging data for the patients recruited in this study it is not feasible for us to have such models. Assuming 
electrostatic, homogeneous, and isotropic tissue properties, we modeled the electrical stimulation field around the electrode as a Gaussian function (FWHM $=1 \mathrm{~mm})$. We discretized this function onto a 3D rectilinear grid $\left(6.3 \times 6.3 \times 1.5 \mathrm{~mm}^{3}\right)$ with $0.1 \mathrm{~mm}$ isotropic cell size centered at the region of the electrode. The grid cells within and on the surface of the electrode were assigned a unit scalar value. Those surrounding cells representing the decreasing stimulation field have their values allocated as indicated by the calculated field strength.

Collection of intra-operative functional data: The intra-operative electrophysiological recording and stimulation data acquired during each procedure were coded in standard form and saved in patient MR image-space. Subsequently the annotated codes of intra-operative functional data were collected to the standard database using the resultant non-rigid transform from the third registration step. The inverse of the deformation grid was then used to map the data in the population-based database from the reference brain template back to an individual brain image.

\subsection{Probabilistic Functional Map Construction}

Two classes of probabilistic maps were generated to describe spatial distribution of population-based actual surgical target stimulation information and deep-brain intraoperative electrophysiological activities respectively.

The target and stimulation information map (Fig. 1) was computed based on the electrode and stimulation field models. First, the 3D grid file representing the electrode and stimulation field created on the post-operative image of each patient was rigidly registered to the pre-operative image-space of the same patient. Then a large rectilinear grid $\left(30 \times 30 \times 20 \mathrm{~mm}^{3}\right)$ with the same cell size was created in the standard brain image-space and the cells were initialized to zero. When the discrete grid file of each patient is non-rigidly mapped to the standard coordinate system, it is superimposed over the large grid. Those grid cells overlapping non-rigid warped patient grid cells have their values incremented accordingly.

Adopting the collection of intra-operative stimulation measurements, we created two electrical stimulation maps $\left(50 \times 50 \times 50 \mathrm{~mm}^{3}\right)$. One map incorporates the functional data relating to suppression of Parkinson's disease (PD) symptoms, while the other integrates data relating to side effects. The map grid cells overlapping the electrical stimulation data from the database have their values assigned and increased according to the stimulation parameters of the functional data. The assigned value is inversely proportional to the stimulation amplitude.

The intra-operative micro-recording data representing the characteristic signals of the STN were integrated into another functional map. A small grid $\left(10 \times 10 \times 10 \mathrm{~mm}^{3}\right)$ covering the STN area of the standard brain template was generated. Since the microrecording data have uniform parameters, the scalar value of each cell in the grid is determined only by the spatial frequency of the data presence.

We adopted Kriging interpolation [10] to generate the final probabilistic functional maps. Kriging, also called optimal spatial linear prediction, is capable of making inferences on unobserved values from data observed at known spatial locations. Kriging interpolation uses those grid cells with scalar values greater than a specified threshold to estimate the possible values for the rest grid cells. The minimum and maximum scalar values on each probabilistic functional map were set to zero and one 
respectively, and the remaining values within the range were normalized accordingly. Maximizing the use of the functional data available in our databases, the final threedimensional probabilistic functional maps consisting of both the actual observed data and the optimal estimation values can be warped to a patient brain prior to surgery to predict the surgical target and trajectory.

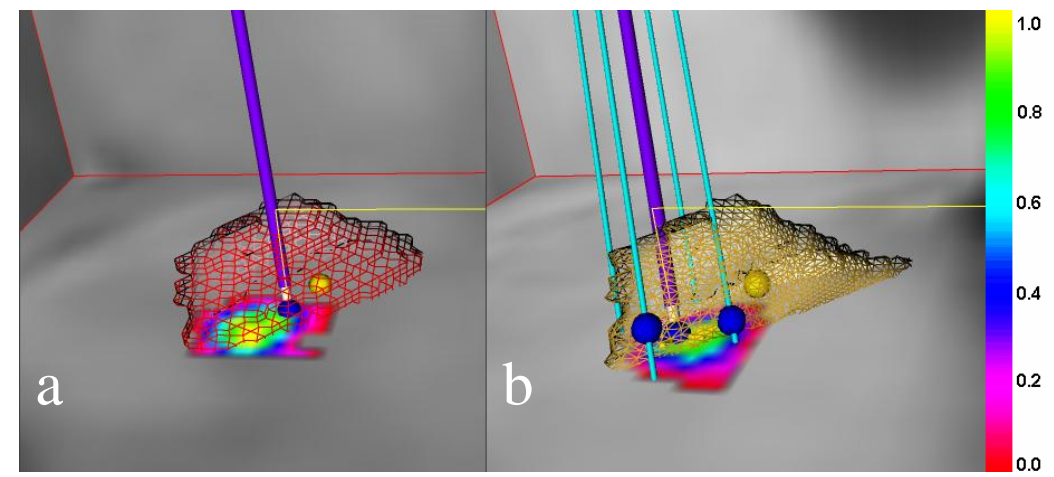

Fig. 1. The standard brain template (left) and a patient brain (right) are displayed by the neurosurgical system. The probabilistic map of surgical target and stimulation information is registered and fused with each image. The color map: probabilistic functional map on which the region in yellow indicates the optimal surgical target location. Dark line: central electrode whose tip is at the actual surgical target position; Bright lines: other four trajectories of "Bengun" electrode assembly; Mesh objects: segmented and registered STN in standard brain and a patient brain space; Bright yellow spheres: centroids of STN.

\section{Clinical Application}

The standard technique practiced by neurosurgeons at our institution for identifying the surgical target includes the combination of direct and indirect targeting strategies. In addition, the trajectory is determined based on the patient's cortical and subcortical anatomy as well as primary vasculature distribution on the pre-operative image to ensure reasonable trajectory entry point and procedure safety. Intra-operatively, the neurosurgeon employs a five-electrode assembly ("Ben-gun") to acquire deep brain electro-physiological information and refine target localization. On the other hand, our neurosurgical planning system [7] estimates the surgical target and trajectory automatically using the probabilistic functional maps described in the previous section. The surgical target location is initially defined as the grid cell with the greatest scalar value in the target and stimulation information map. The average scalar value of grid cells on a trajectory extending $10 \mathrm{~mm}$ above and below this target position at different orientation (lateral-medial: $0^{\circ}-30^{\circ}$ and anterior-posterior: $40^{\circ}-90^{\circ}$ ) is computed. The trajectory with the highest value on the PD symptom relief map and the lowest on the side effect inducing map is considered the optimal trajectory (Fig. 2). Our trajectory identification approach is capable of eliminating any trajectory crossing the lateral ventricle and any sulcus by calculating the image intensity value of each voxel along and around the trajectory on the pre-operative patient brain image. 
Therefore the appropriate entry point can be selected to ensure safety of the surgery. For each of the ten STN DBS cases (5 left and 5 right), we compared the automatically estimated target and trajectory with those identified by the neurosurgeon using standard clinical practice.

\section{Results}

\subsection{Surgical Targeting Accuracy Analysis}

As demonstrated in our previous work [7], stereotactic targeting of the STN using the registered actual surgical target data (centroid of the target cluster) provides less than $2.5 \mathrm{~mm}$ localization error. Therefore we employed the probabilistic functional map containing population-based actual DBS electrode and stimulation field information to predict the surgical target positions. Because of the large sample size for Kriging interpolation, accurate predication of map values at locations where there are no previous patient data available can be accomplished. Table 1 shows that the target locations estimated using this probabilistic map are on average $0.63 \mathrm{~mm}, 0.68 \mathrm{~mm}$, and $0.75 \mathrm{~mm}$ away from the neurosurgeon-determined targets in $\mathrm{x}, \mathrm{y}$, and $\mathrm{z}$ directions respectively for the ten STN DBS procedures, indicating accurate initialization of the surgical target. As distinct from previous targeting techniques [7], this approach employing multiple population-based probabilistic functional maps containing a large number of stimulation field and functional activity data is completely unsupervised and yields higher reproducibility and less variance in target identification.

Table 1. Absolute differences between the probabilistic functional map-initialized and the real surgical targets (x: left-right; $\mathrm{y}$ : posterior-anterior, $\mathrm{z}$ : inferior-superior)

\begin{tabular}{ccccc} 
Difference & $x$ & $y$ & $z$ & $d(x, y, z)$ \\
\hline Avg. $(\mathrm{mm})$ & 0.63 & 0.68 & 0.75 & 1.82 \\
Max $(\mathrm{mm})$ & 1.76 & 1.51 & 1.39 & 3.15 \\
Min $(\mathrm{mm})$ & 0.10 & 0.22 & 0.31 & 0.58 \\
Sd $(\mathrm{mm})$ & 0.67 & 0.52 & 0.44 & 0.77 \\
\hline
\end{tabular}

\subsection{Surgical Trajectory Identification}

A well-planned DBS trajectory ideally encounters stimulation responsive units that reduce tremor, rigidity, and bradykinesia, while avoiding units causing side effects. To identify the optimal surgical trajectory, our neurosurgical system searches the

Table 2. Absolute differences between the trajectory orientation angles estimated with probabilistic functional maps and those of real trajectories

\begin{tabular}{ccc} 
Difference & Anterior-Posterior & Lateral-Medial \\
\hline Avg. $\left({ }^{\circ}\right)$ & 3.1 & 2.3 \\
$\operatorname{Max}\left({ }^{\circ}\right)$ & 4.5 & 4 \\
$\operatorname{Min}\left(\left(^{\circ}\right)\right.$ & 0 & 0 \\
$\operatorname{Sd}\left({ }^{\circ}\right)$ & 1.8 & 1.5 \\
\hline
\end{tabular}


functional map relating to PD symptom relief and that eliciting side effects. The final orientation of the trajectory is determined by the average scalar values of grid cells encountered by the tract $(20 \mathrm{~mm})$ centered at the established optimal surgical target on the two maps. High values on the first map and low values on the second are preferred. Most trajectories are $10^{\circ}$ to $15^{\circ}$ in a lateral-to-medial orientation and $45^{\circ}$ to $70^{\circ}$ anterior-to-posterior. Table 2 reports the absolute difference in the two directions between the optimal trajectories predicted by the probabilistic electrophysiological maps and those identified by the neurosurgeon. In clinical practice, deep brain electrophysiological activity data, used to delineate functional borders and the surgical target, are obtained through only limited number of intra-operative trajectories. However the probabilistic functional maps can take advantage of the population based deep brain measurements. Hence the trajectory estimated with the maps is a result of more comprehensive analysis of electrophysiology.
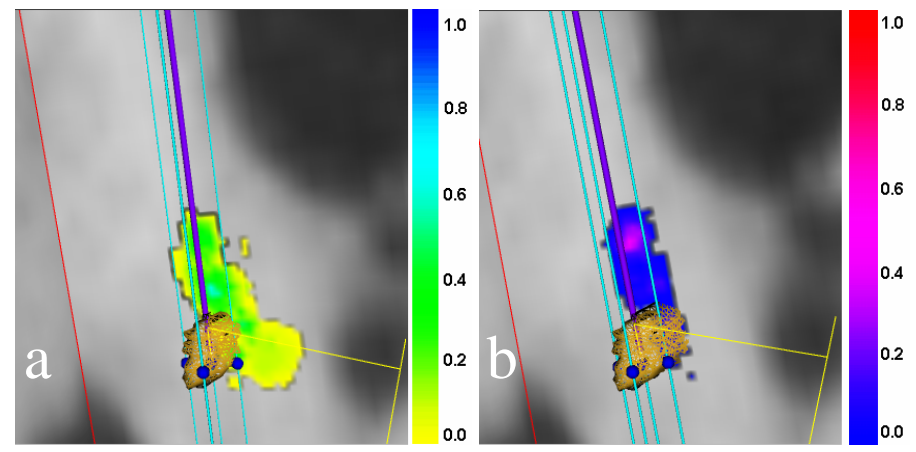

Fig. 2. Left: The functional map relating to PD symptom relief; Right: The functional map relating to side effects; Mesh objects: segmented STN

\subsection{Micro-Recording Data Defined STN}

Although the anterior portion of the $\mathrm{STN}$ can be seen on the $\mathrm{T}_{2}$-weighted images, the distortion observed on $\mathrm{T}_{2}$-weighted images and the lack of visibility of the posterior STN may limit their targeting efficacy. The probabilistic map, generated using the collection of characteristic signals of the STN observed during micro-recording processes, provides a functional representation of the STN. Grid cells with actual or predicted scalar values greater than 0.8 were considered to lie within the functional STN. We compared the centroid location of the functional STN and the real surgical target for each of the ten procedures. As the dorsolateral portion of STN is considered

Table 3. Absolute differences between the centroid locations of functional STN and the real surgical targets (x: left-right; y: posterior-anterior, z: inferior-superior)

\begin{tabular}{ccccc} 
Difference & $x$ & $y$ & $z$ & $d(x, y, z)$ \\
\hline Avg. $(\mathrm{mm})$ & 1.32 & 1.81 & 0.80 & 2.63 \\
Max $(\mathrm{mm})$ & 2.17 & 2.79 & 1.26 & 3.21 \\
$\operatorname{Min}(\mathrm{mm})$ & 0.15 & 0.08 & 0.10 & 0.96 \\
$\operatorname{Sd}(\mathrm{mm})$ & 0.71 & 1.06 & 0.32 & 0.75 \\
\hline
\end{tabular}


the ideal surgical target position at our institution, average absolute difference of 2.63 $\mathrm{mm}$ between the surgical target and the STN centroid is reasonable (Table 3). Their spatial correlation is preserved for 80 percent of the cases.

\section{Discussion}

In this paper, we presented the construction of three-dimensional probabilistic maps of actual surgical target stimulation information and intra-operative functional data. Preliminary studies were conducted to assess the efficacy of target and trajectory prediction for ten STN DBS procedures. The results indicate that the probabilistic functional maps, integrated within our neurosurgical visualization and navigation system [7], facilitate completely automatic identification of surgical target and trajectory for individual patients prior to surgery. We also demonstrated the spatial correlation between the actual surgical target and the centroid of functional STN based on the probabilistic micro-recording data. Constructed with the spatial distribution data of stimulation and electrode contact, another probabilistic STN atlas contributed by Nowinski et al involves a great number of input data [11]. The part of this functional STN with medium and high probabilities correlates well with the anatomical STN derived from the Schaltenbrand-Wahren brain atlas [11]. Our probabilistic functional map for surgical target identification was calculated not only considering the DBS electrode, but also the probable electrical stimulation field. It is necessary to include the electrical stimulation information, since axons within $2.5 \mathrm{~mm}$ from the center of the electrode may be activated by monopolar stimulation [12]. Some studies demonstrated that brain shift and deformation caused by cerebrospinal fluid (CSF) leak occurring during DBS surgery around the functional target region is trivial $(<1 \mathrm{~mm})$ and does not affect the accuracy of target localization performed on pre-operative image space [13]. However, a more recent work by Pollo et al. [14] revealed displacement of the electrode in the distal direction that may be due to the patient position change, CSF leakage, and cannula and /or electrode insertion during image acquisition and surgical procedure. This issue should be further studied to quantify the influence of each contributive factor and eventually correct the possible brain shift. Three-dimensional probabilistic functional maps containing the actual intra-operatively obtained data at known locations and optimally predicted data generated by Kriging interpolation at the remaining areas enable comprehensive quantitative analysis of the surgical target and trajectory. The neurosurgical system saves target location, trajectory orientation, and the average scalar values of grid cells encountered by the trajectory centered at the optimal surgical target on the functional maps in a text file for additional evaluation. Although the system incorporating the probabilistic functional maps makes fully automatic surgical target and trajectory estimation feasible, thorough validation studies must nevertheless be carried out within a clinical context. In our ongoing studies we will obtain clinical information, analyze the correlation between the surgical target location and the surgical outcome, and re-present our previously conducted analysis with respect to the actual clinical evaluation data. Once this automatic surgical target and trajectory identification technique is clinically validated, the neurosurgeon at our institution would employ it for the planning and guidance of DBS procedures. 
Acknowledgements. The authors acknowledge the financial support from CIHR, ORDCF, CFI, and OIT.

\section{References}

1. Machado, A., Rezai, A.R., Kopell, B.H., Gross, R.E., Sharan, A.D., Benabid, A.L.: Deep Brain Stimulation for Parkinson's Disease: Surgical Technique and Perioperative Management. Mov. Disord. 21(suppl. 14), S247-S258 (2006)

2. Halpern, C., Hurtig, H., Jaggi, J., Grossman, M., Won, M., Baltuch, G.: Deep brain stimulation in neurologic disorders. Parkinsonism Relat. Disord. 13, 1-16 (2007)

3. Ganser, K.A., Dickhaus, H., Metzner, R., Wirtz, C.R.: A deformable digital brain atlas system according to Talairach and Tournoux. Med. Imag. Analy. 8, 3-22 (2004)

4. Yelnik, J., Bardinet, E., Dormont, D., Malandain, G., Ourselin, S., Tande, D., Karachi, C., Ayache, N., Cornu, P., Agid, Y.: A three-dimensional, histological and deformable atlas of the human basal ganglia. I. Atlas construction based on immunohistochemical and MRI data. NeuroImage 34, 618-638 (2007)

5. D'Haese, P.F., Cetinkaya, E., Konrad, P.E., Kao, C., Dawant, B.M.: Computer-aided placement of deep brain stimulators: from planning to intraoperative guidance. IEEE Trans. Med. Imag. 24(11), 1469-1478 (2005)

6. Chakravarty, M.M., Sadikot, A.F., Mongia, S., Bertrand, G., Collins, D.L.: Towards a multi-modal atlas for neurosurgical planning. In: Larsen, R., Nielsen, M., Sporring, J. (eds.) MICCAI 2006. LNCS, vol. 4191, pp. 389-396. Springer, Heidelberg (2006)

7. Guo, T., Finnis, K.W., Deoni, S.C.L., Parrent, A.G., Peters, T.M.: Comparison of different targeting methods for subthalamic nucleus deep brain stimulation. In: Larsen, R., Nielsen, M., Sporring, J. (eds.) MICCAI 2006. LNCS, vol. 4190, pp. 768-775. Springer, Heidelberg (2006)

8. Guo, T., Starreveld, Y.P., Peters, T.M.: Evaluation and validation methods for intersubject non-rigid 3D image registration of the human brain. Proc. SPIE Medical Imaging 5744, 594-603 (2005)

9. Butson, C.R., Cooper, S.E., Henderson, J.M., McIntyre, C.C.: Patient-specific analysis of the volume of tissue activated during deep brain stimulation. NeuroImage 34, 661-670 (2007)

10. Cressie, N.A.C.: Statistics for Spatial Data. A Wiley-Interscience Publication, Chichester (1991)

11. Nowinski, W.L., Thirunavuukarasuu, A., Liu, J., Benabid, A.L.: Correlation between the anatomical and functional human subthalamic nucleus. Stereotact. Funct. Neurosurg. 85, 88-93 (2007)

12. Wu, Y.R., Levy, R., Ashby, P., Tasker, R.R., Dostrovsky, J.O.: Does stimulation of the GPi control dyskinesia by activating inhibitory axons. Mov. Disord. 16, 208-216 (2001)

13. Bardinet, É., Cathier, P., Roche, A., Ayache, N., Dormont, D.: A Posteriori Validation of Pre-operative Planning in Functional Neurosurgery by Quantification of Brain Pneumocephalus. In: Dohi, T., Kikinis, R. (eds.) MICCAI 2002. LNCS, vol. 2488, pp. 323-330. Springer, Heidelberg (2002)

14. Pollo, C., Vingerhoets, F., Pralong, E., Ghika, J., Makder, P., Meuli, R., Thiran, J.P., Villemure, J.G.: Localization of electrodes in the subthalamic nucleus on magnetic resonance imaging. J. Neurosurg. 106, 36-44 (2007) 